\title{
Reduced glycaemic and insulinaemic responses following trehalose and isomaltulose ingestion: implications for postprandial substrate use in impaired glucose-tolerant subjects
}

\author{
Judith G. P. van Can ${ }^{1 *}$, Luc J. C. van Loon ${ }^{2}$, Fred Brouns ${ }^{1,3}$ and Ellen E. Blaak ${ }^{1}$ \\ ${ }^{1}$ Department of Human Biology, Maastricht University Medical Centre, Universiteitssingel 50, 6229 ER Maastricht, \\ The Netherlands \\ ${ }^{2}$ Department of Human Movement Sciences, NUTRIM School for Nutrition, Toxicology and Metabolism, Maastricht University \\ Medical Centre, Maastricht, The Netherlands \\ ${ }^{3}$ Cargill RED Center, Vilvoorde, Belgium \\ (Submitted 11 May 2011 - Final revision received 18 October 2011 - Accepted 8 November 2011 - First published online 15 December 2011)
}

\begin{abstract}
The impact of slowly digestible sugars in reducing the risk of developing obesity and related metabolic disorders remains unclear. We hypothesised that such carbohydrates $(\mathrm{CHO})$, resulting in a lower glycaemic and insulinaemic response, may lead to greater postprandial fat oxidation rates in subjects with impaired glucose tolerance (IGT). The present study intends to compare the postprandial metabolic responses to the ingestion of glucose (GLUC) $v$. trehalose (TRE) and sucrose (SUC) $v$. isomaltulose (IMU). In a randomised, singleblind, cross-over design, ten overweight IGT subjects were studied four times, following ingestion of different CHO drinks either at breakfast or in combination with a mixed meal at lunch. Before and $3 \mathrm{~h}$ after $\mathrm{CHO}$ ingestion, energy expenditure, substrate utilisation and circulating metabolite concentrations were determined. Ingestion of $\mathrm{CHO}$ drinks with a meal resulted in an attenuated rise in GLUC $(-33 \%)$ and insulin $(-14 \%)$ concentrations following TRE when compared with GLUC and following IMU, an attenuation of 43 and $34 \%$ when compared with SUC ingestion, respectively. Additionally, there was less inhibition of the rise in NEFA concentrations and less decline in postprandial fat oxidation (22\%) after IMU when compared with SUC, whereas TRE did not differ from GLUC. The attenuated rise in GLUC and insulin concentrations following IMU ingestion attenuated the postprandial inhibition of fat oxidation compared with SUC when co-ingested with a meal. This suggests that exchange of SUC in the diet for IMU may result in a more favourable metabolic response and may help to reduce the risks associated with obesity and type 2 diabetes.
\end{abstract}

\section{Key words: Trehalose: Isomaltulose: Substrate use}

The increasing prevalence of obesity and obesity-related disorders such as type 2 diabetes has become the greatest health problem of the present and coming decades ${ }^{(1)}$. According to the physiological state where abnormalities in glucose (GLUC) metabolism are present but below the cut-off point for the diagnosis of type 2 diabetes, individuals can be grouped into those who suffer from (1) impaired fasting GLUC or (2) impaired GLUC tolerance (IGT). Individuals with isolated IGT show moderate to severe muscle insulin resistance and suffer from a defect in both the early- and late-phase insulin secretory response to an oral GLUC load. Patients with IGT have a 2- to 5-fold greater risk of developing CVD, compared with age-matched normoglycaemic controls $^{(2)}$. Each year, about $10 \%$ of the subjects with impaired fasting GLUC and IGT progress to develop type 2 diabetes ${ }^{(3)}$. Lifestyle intervention, directed towards a healthy diet, i.e. a reduction in saturated fat intake and an increase in lowglycaemic carbohydrate (CHO) intake, and an increase in habitual physical activity level, has proven effective in preventing or delaying the onset of type 2 diabetes in subjects with $\mathrm{IGT}^{(4,5)}$. Interventions to reduce the glycaemic index (GI) and glycaemic load of the daily diet have received much interest in nutritional research ${ }^{(6,7)}$. So far, numerous studies have reported that diets low in GI or glycaemic load can have beneficial effects on weight loss and/or reduce the risk of developing chronic metabolic disease in human subjects ${ }^{(6,8-10)}$. Whereas some have suggested that diets high in $\mathrm{CHO}$ may have an adverse effect on TAG concentrations

Abbreviations: CHO, carbohydrate; En\%, percentage of energy; GI, glycaemic index; GLUC, glucose; iAUC, incremental area under the curve; IGT, impaired glucose tolerance; IMU, isomaltulose; SUC, sucrose; TRE, trehalose.

*Corresponding author: J. G. P. van Can, fax +31 43 3670976, email j.vancan@maastrichtuniversity.nl 
and HDL-cholesterol ${ }^{(11)}$, others have failed to confirm those findings. The apparent discrepancy between studies is probably attributed to differences in the duration of the intervention, sex and the use of different types of sugars between studies $^{(12-14)}$.

It has been hypothesised that low-GI foods may affect body-weight control and insulin sensitivity by promoting satiety and stimulating fat oxidation at the expense of $\mathrm{CHO}$ oxidation $^{(15)}$. This increased fat oxidation may reduce fat storage in adipose and non-adipose tissues, thereby promoting insulin sensitivity and an improved metabolic profile. Indeed, animal studies have shown that a reduced GI can shift substrate use in favour of fat oxidation, independent of diet-induced changes in body composition or energy intake $^{(16-18)}$. We recently showed that a reduced glycaemic response after a mixed meal containing trehalose (TRE) or isomaltulose (IMU) may improve fat oxidation rates at the expense of $\mathrm{CHO}$ oxidation in overweight subjects ${ }^{(19,20)}$. Similar findings ${ }^{(21)}$ have also been observed during exercise conditions.

So far, it is not known whether these beneficial effects on fat oxidation also extend to impaired GLUC-tolerant subjects who show profound disturbances in the capacity to utilise fat as a substrate source during basal fasting conditions as well as in the capacity to switch between $\mathrm{CHO}$ and fat oxidation during postprandial conditions ${ }^{(22)}$. The fact that disturbances in fatty acid uptake and oxidation are already present in the pre-diabetic state suggests a key role in the progression towards type 2 diabetes ${ }^{(23)}$. Consequently, more work is warranted to assess the impact of low-GI $\mathrm{CHO}$ on postprandial substrate use in an obese group with IGT. Therefore, we examined the metabolic response to the ingestion of two slowly digestible $\mathrm{CHO}$ sources, TRE and IMU, respectively. TRE is a GLUC disaccharide with an $\alpha-1,1$ glycoside linkage, whereas IMU is a disaccharide produced by an enzymatic conversion of sucrose (SUC).

We hypothesised that the ingestion of TRE and IMU will be accompanied by a lower glycaemic and/or insulinaemic response, an attenuated inhibition of postprandial lipolysis and fat oxidation rate and a lower plasma TAG response when compared with GLUC and SUC, respectively.

\section{Methods \\ Subjects}

A total of ten overweight men ( $n$ 6) and women $(n$ 4), of which two were post-menopausal, with IGT were recruited for the present study. Subjects' characteristics are presented in Table 1 . Subjects with type 2 diabetes and/or overt cardiovascular complications, and those using medication for digestive disorders were excluded from the study. All subjects were screened with a standard $75 \mathrm{~g}$ oral glucose tolerance test after an overnight fast. IGT was diagnosed based on the WHO criteria. The study was conducted according to the guidelines laid down in the Declaration of Helsinki and all procedures involving human subjects/patients were approved by the Medical Ethical Committee of the Maastricht
Table 1. Subjects' characteristics

(Mean values and standard deviations)

\begin{tabular}{|c|c|c|}
\hline & \multicolumn{2}{|c|}{ Subjects (female $n 4$, male $n 6$ ) } \\
\hline & Mean & SD \\
\hline Age (years) & 56 & 8 \\
\hline Weight (kg) & $91 \cdot 3$ & $20 \cdot 3$ \\
\hline BMI $\left(\mathrm{kg} / \mathrm{m}^{2}\right)$ & $30 \cdot 8$ & 4.9 \\
\hline Fasting glucose $(\mathrm{mmol} / \mathrm{l})$ & $5 \cdot 63$ & 0.64 \\
\hline $2 \mathrm{~h}$ Glucose $(\mathrm{mmol} / \mathrm{l})$ & $8 \cdot 78$ & 0.96 \\
\hline Fasting insulin $(\mu \mathrm{U} / \mathrm{ml})^{\star}$ & 18 & 8.9 \\
\hline HOMA-IR & 4.63 & 2.53 \\
\hline $\mathrm{HbA}_{1 \mathrm{c}}(\%)$ & 5.85 & 0.19 \\
\hline Fasting NEFA $(\mu \mathrm{mol} / \mathrm{l})$ & 402 & 101 \\
\hline Fasting TAG (mmol/l) & $1 \cdot 15$ & 0.47 \\
\hline ALAT (U/I) & 30 & $9 \cdot 7$ \\
\hline Creatinine $(\mu \mathrm{mol} / \mathrm{l})$ & 79 & $15 \cdot 2$ \\
\hline
\end{tabular}

HOMA-IR; homeostasis model assessment-insulin resistance; $\mathrm{HbA}_{1 \mathrm{c}}$, glycated $\mathrm{Hb}$; ALAT, alanine transferase.

*To convert insulin from $\mu \mathrm{U} / \mathrm{ml}$ to pmol/l, multiply by 6 .

University Medical Centre. All subjects gave written informed consent.

\section{Study design}

Each subject participated in four trials, separated by a 1-week washout period, in which the metabolic response was measured after ingestion of four different $\mathrm{CHO}$ drinks. $\mathrm{CHO}$ drinks were ingested after an overnight fast (breakfast drink) and in combination with a standardised mixed meal (lunch). The CHO drinks (GLUC, TRE, SUC and IMU) were provided in a single-blind, randomised order.

\section{Protocol}

At the beginning of the experimental day, after an overnight fast, a cannula was inserted into an antecubital vein. The $\mathrm{CHO}$ load consisted of $75 \mathrm{~g}$ CHO equivalents and was dissolved in $400 \mathrm{ml}$ of water. The $\mathrm{CHO}$ drink was consumed after an overnight fast at breakfast (08.45 hours) or in combination with a mixed meal at lunch (12.30 hours) within a period of $15 \mathrm{~min}$. Energy expenditure and substrate utilisation were measured, before and for $3 \mathrm{~h}$ after ingestion of the meal and/or drink using a ventilated hood system (Omnical) ${ }^{(24)}$. Gas analyses, recorded every minute, were performed by dual paramagnetic $\mathrm{O}_{2}$ analysers and dual $\mathrm{IR} \mathrm{CO}_{2}$ analysers (type 1156, 1507, 1520; Servomex), similar to the analysis system described by Schoffelen et al. ${ }^{(25)}$. Blood samples were taken before consumption of the meal/drinks $(t=-5 \mathrm{~min})$ and then at $t=30,60,90,120,150$ and $180 \mathrm{~min}$ after $\mathrm{CHO}$ ingestion to determine circulating metabolites and hormone concentrations. Expired breath samples were collected each hour to determine ${ }^{13} \mathrm{CO}_{2}$ enrichment. Energy expenditure and substrate use were calculated using the formulas of Weir ${ }^{(26)}$ and Frayn ${ }^{(27)}$.

Lunch had a total energy content equivalent of $50 \%$ of calculated $24 \mathrm{~h}$ resting energy expenditure based upon the formula of Harris \& Benedict ${ }^{(28)}$. Lunch macronutrient composition 
represented $55 \mathrm{En} \% \mathrm{CHO}, 30 \mathrm{En} \%$ fat and $15 \mathrm{En} \%$ protein; $25 \mathrm{En} \%$ of the total energy content of the meal was provided in the form of a beverage containing either TRE, IMU, GLUC or SUC.

\section{Test products}

Trehalose. TRE is a disaccharide of GLUC with an $\alpha-1,1$ glycoside linkage. It is a non-reducing sugar that is naturally present in honey, bread, mushrooms and fermented drinks. For our experiment, ${ }^{13} \mathrm{C}$-enriched TRE was produced by enzymatic conversion using maize starch as the base material. In the human intestine, TRE is exclusively digested by epithelial trehalase into two D-GLUC molecules, which are subsequently absorbed and metabolised ${ }^{(29,30)}$. Apart from the trehalase action, it appears that ingestion, hydrolysis, absorption and metabolism of TRE are essentially identical to all other digestible disaccharides ${ }^{(29)}$.

Isomaltulose. IMU is a disaccharide produced by an enzymatic conversion of SUC, whereby the 1,2-glycosidic linkage between GLUC and fructose is rearranged to a 1,6-glycosidic linkage. For our experiment, ${ }^{13} \mathrm{C}$-enriched IMU was produced by enzymatic conversion using cane sugar as the base material. The sucrase-isomaltase complex located on the brush-border membrane of the small-intestinal epithelial cells hydrolyses both IMU and SUC. The resulting monosaccharides, GLUC and fructose, are taken up into the portal blood ${ }^{(31)}$.

\section{Biochemical analyses}

At all time points, $8 \mathrm{ml}$ blood were collected in pre-chilled tubes with $200 \mu \mathrm{l}$ of $0 \cdot 2 \mathrm{M}$-EDTA (Sigma). After collection, blood samples were centrifuged immediately at $4^{\circ} \mathrm{C}$ for $10 \mathrm{~min}$ at $1000 \mathrm{~g}$ and frozen at $-80^{\circ} \mathrm{C}$ until further analysis. Plasma was used for the enzymatic colorimetric quantification of NEFA (NEFA C kit; Wako Chemicals) and TAG (Sigma) on a COBAS FARA centrifugal spectrophotometer (Roche Diagnostica). Plasma GLUC concentration (ABX Diagnostics) was measured enzymatically on a COBAS MIRA automated spectrophotometer (Roche Diagnostica). Plasma insulin was measured with a double antibody RIA (Linco Research). Breath samples were analysed for ${ }^{13} \mathrm{C}:{ }^{12} \mathrm{C}$ ratio by $\mathrm{GC}$-isotope ratio MS (Finnigan MAT 252; Finnigan), as described in van Can et al. ${ }^{(19,20)}$.

\section{Statistics}

A computerised statistics program (SPSS 15 for Windows; SPSS, Inc.) was used to perform all calculations. All data are expressed as means with their standard errors. The total response of parameters after $\mathrm{CHO}$ ingestion was expressed as the incremental area under the curve (iAUC) and calculated by the trapezoid method. Response is defined in the Results section as iAUC, unless mentioned otherwise. Differences between responses to GLUC $v$. TRE and SUC $v$. IMU were analysed by means of Student's paired $t$ test. Student's paired $t$ test was used to compare differences in peak response between the different $\mathrm{CHO}$. The four $\mathrm{CHO}$ were not compared with each other due to the fact that they are made out of different CHO sources. Therefore, TRE is compared with GLUC and IMU compared with SUC.

\section{Results}

\section{Circulating metabolites}

Glucose response. Ingestion of TRE resulted in lower peak GLUC concentrations when compared with GLUC both during breakfast drinks $(P<0 \cdot 01)$ and lunch $(P=0 \cdot 001)$ (Fig. 1(a)). This did, however, not result in a significant difference in glycaemic response, expressed as iAUC (Table 2). GLUC peaks were lower after ingestion of IMU compared with SUC during breakfast $(P=0 \cdot 01)$ and lunch $(P=0 \cdot 001)$ (Fig. 1(b)). There was a reduced incremental glycaemic response after the ingestion of IMU when combined with a mixed meal $(P<0 \cdot 001$; Table 3$)$.

Insulin response. TRE resulted in lower peak insulin concentrations when compared with GLUC following breakfast $(P=0.003)$ and lunch $(P=0.025$; Fig. $1(\mathrm{c}))$. The iAUC was lower after the ingestion of TRE compared with GLUC during breakfast $(P=0.009)$ but not when TRE was ingested with a mixed meal during lunch (Table 2). Insulin responses were reduced after the ingestion of IMU compared with SUC following breakfast (iAUC, $P<0.05$ ) and lunch (iAUC, $P=0.001)$ (Fig. 1(d); Table 3).

NEFA response. As expected, plasma NEFA concentrations decreased after $\mathrm{CHO}$ ingestion. Ingestion of either TRE or GLUC resulted in a similar NEFA response pattern, also when ingested in combination with a mixed meal (Fig. 1(e)). There were no significant differences in the integrated decrement between TRE and GLUC (Table 2). Ingestion of IMU in combination with a mixed meal during lunch resulted in a less inhibition of the decline in plasma NEFA concentrations when compared with SUC ( $P<0 \cdot 0001$; Fig. 1(f); Table 3).

TAG response. TAG concentrations increased after the ingestion of the different $\mathrm{CHO}$ drinks and when the drinks were ingested in combination with a mixed meal. There were no differences in incremental TAG AUC after the ingestion of TRE compared with GLUC during breakfast and lunch (Fig. 2(a); Table 2). There was a trend towards a lower iAUC when IMU was ingested in combination with a mixed meal ( $P=0 \cdot 06$; Fig. 2(b); Table 3 ).

\section{Thermogenesis and substrate oxidation}

There were no differences in the thermogenic response between the $\mathrm{CHO}$ drinks during breakfast or when ingested with a mixed meal (Tables 2 and 3).

There were no differences in the iAUC of the respiratory quotient after TRE ingestion compared with GLUC during breakfast and lunch (Table 2). Intake of IMU did not result in differences in respiratory quotient response compared with SUC during breakfast, whereas IMU ingested in combination with a mixed meal resulted in a reduced respiratory quotient response compared with SUC ( $P=0 \cdot 034$; Table 3$)$.

There were no significant differences in the decrement in fat oxidation rates between TRE and GLUC during breakfast and lunch (Fig. 2(c); Table 2). Fat oxidation did not differ between IMU and SUC during breakfast; interestingly, fat oxidation was significantly less suppressed after IMU when compared with SUC following lunch $(P<0 \cdot 05$; Fig. 2(d); Table 3$)$. 
There were no significant differences in $\mathrm{CHO}$ oxidation between TRE and GLUC during breakfast and lunch (Fig. 2(e), Table 2). Intake of IMU did not result in significant differences following breakfast when compared with SUC, whereas the increment in $\mathrm{CHO}$ oxidation was lower after the ingestion of IMU when compared with SUC during lunch ( $P=0.036$; Fig. 2(f); Table 3).

No differences were observed in the minimal estimates of exogenous $\mathrm{CHO}$ oxidation rates between the experiments. The mean percentage of the enriched $\mathrm{CHO}$ oxidised, as (a)

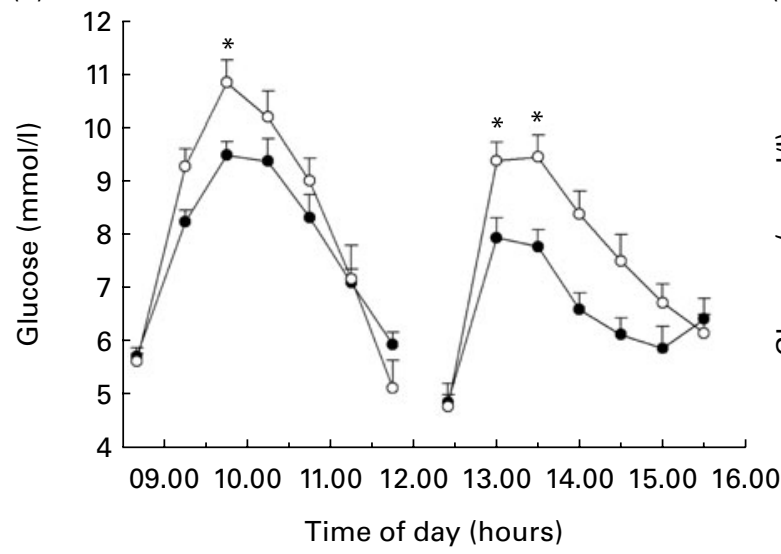

(b)

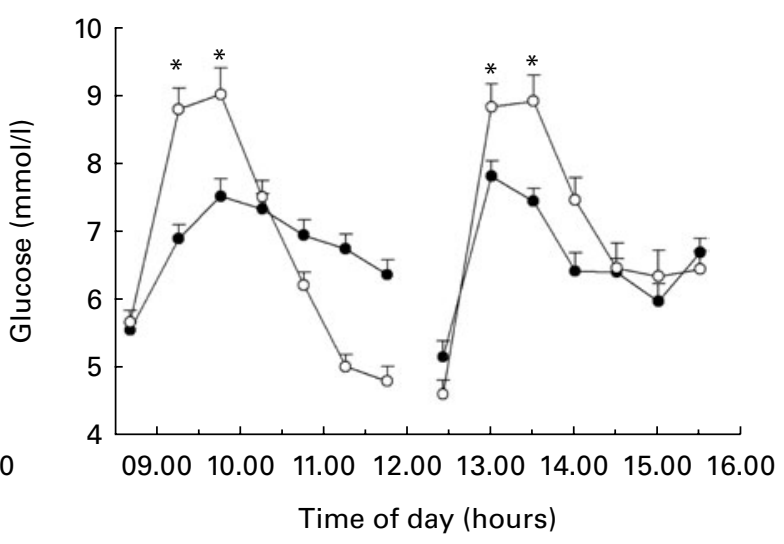

(c)

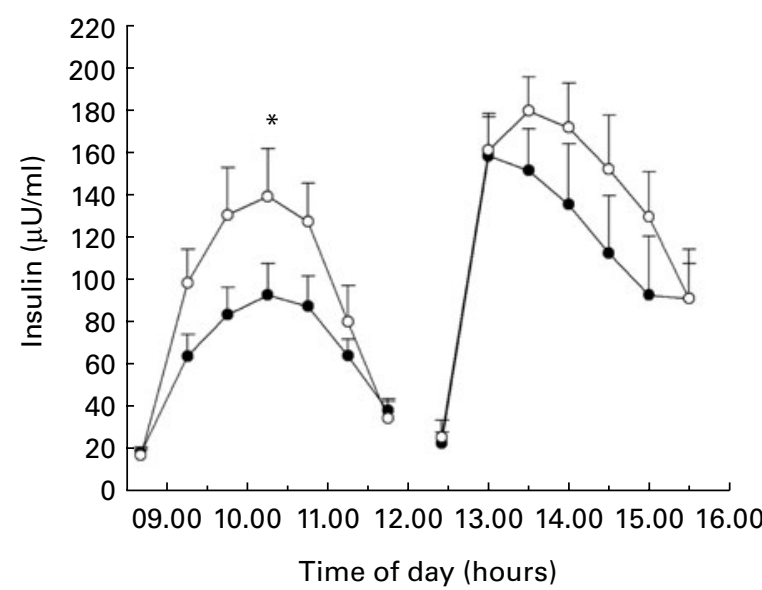

(e)

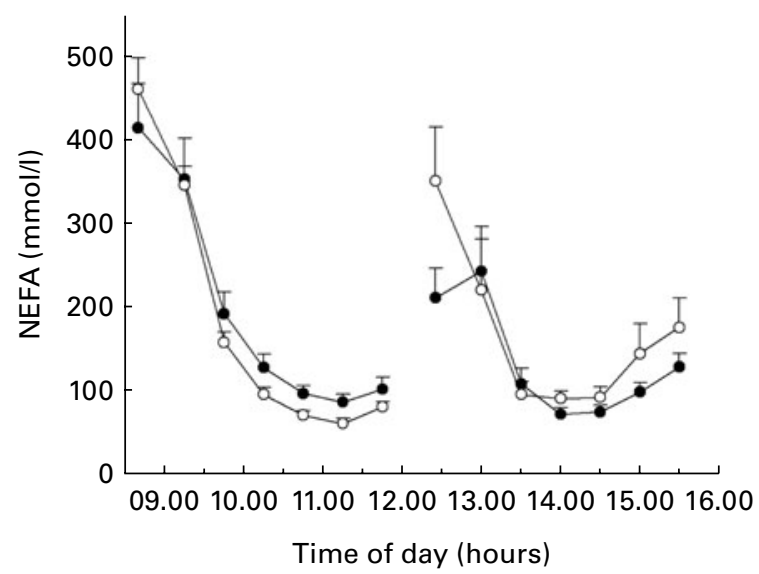

(d)

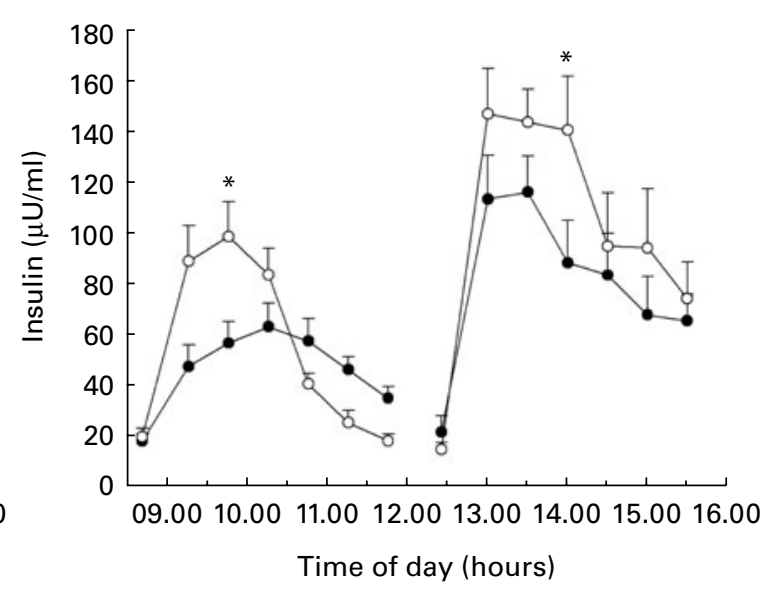

(f)

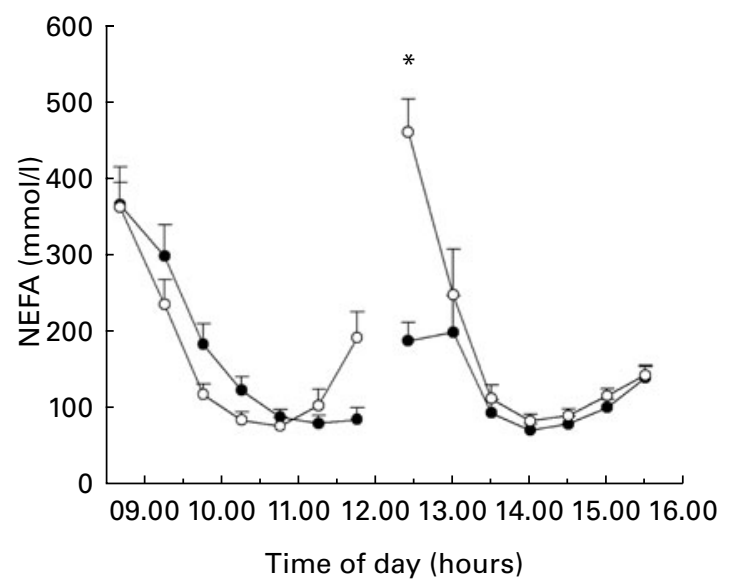

Fig. 1. Time course of the glycaemic response after the intake of (a) trehalose (TRE, $-\bullet-) v$. glucose (GLUC, $-0-$ ) and (b) isomaltulose (IMU, $-\bullet-) v$. sucrose (SUC, -O-). Time course of the insulinaemic response after the intake of (c) TRE $v$. GLUC and (d) IMU $v$. SUC. To convert insulin from $\mu U / \mathrm{ml}$ to $\mathrm{pmol} / \mathrm{l}$, multiply by 6 . Time course of NEFA concentrations after the intake of (e) TRE $v$. GLUC and (f) IMU $v$. SUC. Values are means, with standard errors of the mean represented by vertical bars $(n 10)$. ${ }^{*}$ Mean values were significantly different $(P<0.05)$. 
Table 2. Metabolic responses, expressed as change in area under the curve (iAUC), after ingestion of trehalose and glucose

\begin{tabular}{|c|c|c|c|c|}
\hline & \multicolumn{2}{|c|}{ iAUC breakfast over $3 \mathrm{~h}$} & \multicolumn{2}{|c|}{ iAUC lunch over $3 \mathrm{~h}$} \\
\hline & Trehalose & Glucose & Trehalose & Glucose \\
\hline Glucose $(\mathrm{mmol} / \mathrm{l}$ over $3 \mathrm{~h})$ & 428 & 554 & 374 & 559 \\
\hline Insulin $(\mu \mathrm{U} / \mathrm{ml}$ over $3 \mathrm{~h}) \dagger$ & $9425^{\star \star}$ & 15216 & 17934 & 20875 \\
\hline NEFA $(\mathrm{mmol} / \mathrm{l}$ over $3 \mathrm{~h})$ & -41527 & -52838 & -15200 & -55284 \\
\hline TAG $(\mathrm{mmol} / /$ over $3 \mathrm{~h})$ & 13182 & 9158 & 56566 & 6863 \\
\hline Fat oxidation (g over $3 \mathrm{~h}$ ) & -1.32 & $-2 \cdot 66$ & 0.62 & 0.38 \\
\hline Carbohydrate oxidation (g over $3 \mathrm{~h}$ ) & $6 \cdot 55$ & $10 \cdot 11$ & 8.72 & $9 \cdot 6$ \\
\hline Energy expenditure (kJ over $3 \mathrm{~h}$ ) & 33 & 53 & 144 & 166 \\
\hline Respiratory quotient (over $3 \mathrm{~h}$ ) & 5.47 & $7 \cdot 60$ & $1 \cdot 77$ & 2.68 \\
\hline
\end{tabular}

Mean value was significantly different from that of glucose: ${ }^{* \star} P<0.01$.

$\dagger$ To convert insulin from $\mu \mathrm{U} / \mathrm{ml}$ to pmol/l, multiply by 6 .

calculated by the recovery of ${ }^{13} \mathrm{CO}_{2}$ in the expired breath, was $11 \%$ for TRE, $12 \%$ for GLUC, $15 \%$ for IMU and $19 \%$ for SUC, respectively.

\section{Discussion}

\section{Substrate utilisation}

The main finding of the present study is that intake of IMU in combination with a mixed meal resulted in an attenuated rise in postprandial plasma GLUC and insulin concentrations and a lesser inhibition of circulating NEFA concentration and fat oxidation compared with SUC ingestion. The reduced inhibition of postprandial fat oxidation could be attributed to a greater supply of NEFA to the fat-oxidising tissue, secondary to a reduced insulin-mediated suppression of lipolysis ${ }^{(32)}$. The present results seem consistent with other work, highlighting the stimulating effects of IMU ingestion on postprandial fat oxidation and/or lipid deposition when compared with SUC, in rats, healthy and overweight subjects ${ }^{(19,33,34)}$. The present study shows that IMU ingestion in exchange for SUC has beneficial effects in subjects with IGT and, as such, may help to prevent the progression into type 2 diabetes.

The attenuated postprandial decline in fat oxidation induced by the ingestion of IMU may have implications for body-weight control. Flatt ${ }^{(35)}$ proposed that subjects who continue to oxidise $\mathrm{CHO}$ in the post-absorptive state deplete their endogenous glycogen stores, thereby stimulating food intake.
Through this mechanism, inter-individual differences in substrate selection may play a key role in the development of obesity. A lower decrement in circulating NEFA and fat oxidation following the ingestion of more slowly digestible $\mathrm{CHO}$ may favour fat oxidation above storage, resulting in less fat accumulation in non-adipose tissues with a favourable effect on insulin sensitivity by preventing late hypoglycaemia and the accompanying increase in plasma NEFA concentrations ${ }^{(36)}$. High NEFA concentrations may be linked with insulin resistance and CVD by increasing muscle ectopic fat promoting lipotoxicity, which may reduce insulin action ${ }^{(37)}$.

\section{Glycaemic and insulinaemic responses}

The attenuated glycaemic and insulinaemic responses following TRE and IMU ingestion are attributed to the slower rates at which TRE and IMU are digested and absorbed. Several studies have shown that the absorption rates of TRE and IMU are slower than GLUC and SUC, respectively ${ }^{(38,39)}$. TRE as well as IMU are absorbed and tolerated well in human subjects $^{(29,31)}$. Reduced GLUC and insulin concentrations after the intake of TRE or IMU have been observed in trained athletes, healthy subjects, as well as in overweight subjects ${ }^{(19,20,40,41)}$. The present study is the first to show that intake of TRE and IMU attenuated the postprandial rise in plasma GLUC and insulin concentrations in subjects with IGT. Although there were no significant differences in the integrated glycaemic

Table 3. Metabolic responses, expressed as change in area under the curve (iAUC), after ingestion of isomaltulose and sucrose

\begin{tabular}{|c|c|c|c|c|}
\hline & \multicolumn{2}{|c|}{ iAUC breakfast over $3 \mathrm{~h}$} & \multicolumn{2}{|c|}{ iAUC lunch over $3 \mathrm{~h}$} \\
\hline & Isomaltulose & Sucrose & Isomaltulose & Sucrose \\
\hline Glucose $(\mathrm{mmol} / \mathrm{l}$ over $3 \mathrm{~h})$ & 248 & 266 & $279^{\star \star}$ & 489 \\
\hline Insulin $(\mu \mathrm{U} / \mathrm{ml}$ over $3 \mathrm{~h}) \dagger$ & $5779^{\star}$ & 7326 & $11726^{\star \star}$ & 17658 \\
\hline NEFA (mmol/l over $3 \mathrm{~h})$ & -36137 & -38869 & $-12880^{\star *}$ & -55284 \\
\hline TAG $(\mathrm{mmol} / \mathrm{l}$ over $3 \mathrm{~h})$ & 19802 & 20896 & 40787 & 60680 \\
\hline Fat oxidation (g over $3 \mathrm{~h}$ ) & -4.22 & $-5 \cdot 20$ & $-0.89^{\star}$ & -4.08 \\
\hline Carbohydrate oxidation (g over $3 \mathrm{~h}$ ) & $14 \cdot 14$ & $16 \cdot 19$ & $12 \cdot 27^{\star}$ & $23 \cdot 16$ \\
\hline Energy expenditure (kJ over $3 \mathrm{~h}$ ) & 54 & 55 & 156 & 158 \\
\hline Respiratory quotient (over $3 \mathrm{~h}$ ) & $11 \cdot 14$ & $13 \cdot 18$ & $4.89^{*}$ & $12 \cdot 24$ \\
\hline
\end{tabular}

Mean values were significantly different from those of sucrose: ${ }^{\star} P<0.05,{ }^{\star \star} P<0.01$. $\dagger$ To convert insulin from $\mu \mathrm{U} / \mathrm{ml}$ to pmol/l, multiply by 6 . 
(a)

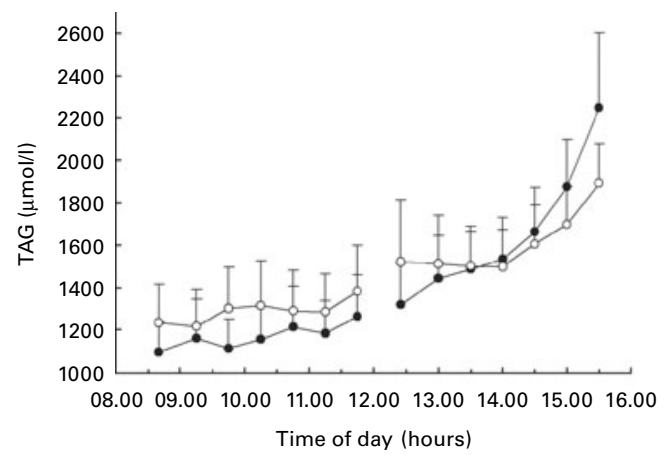

(c)

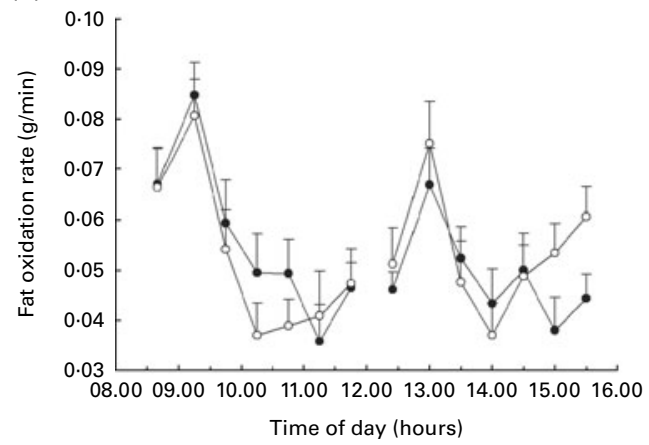

(e)

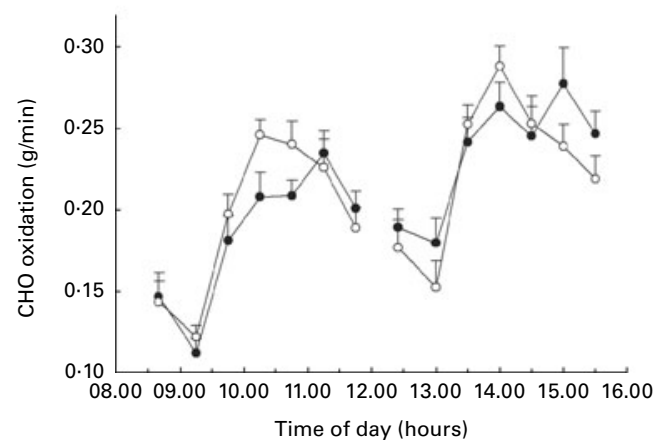

(b)

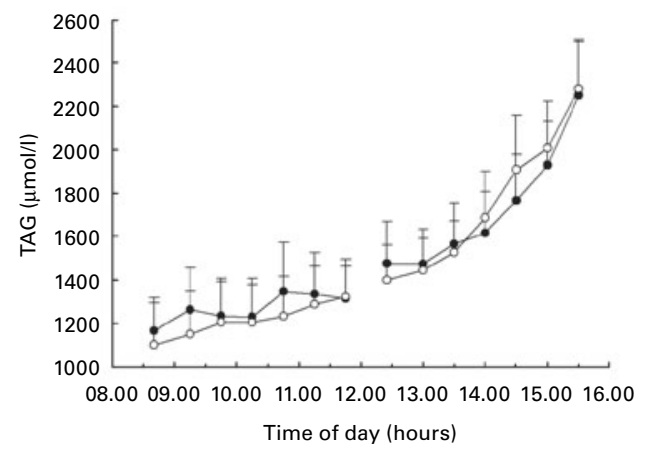

(d)

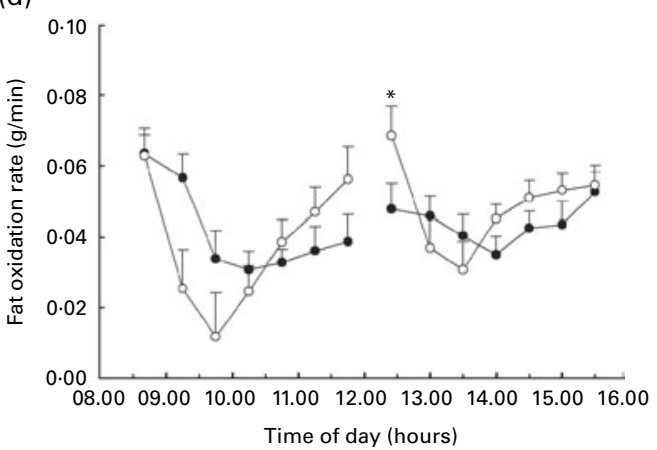

(f)

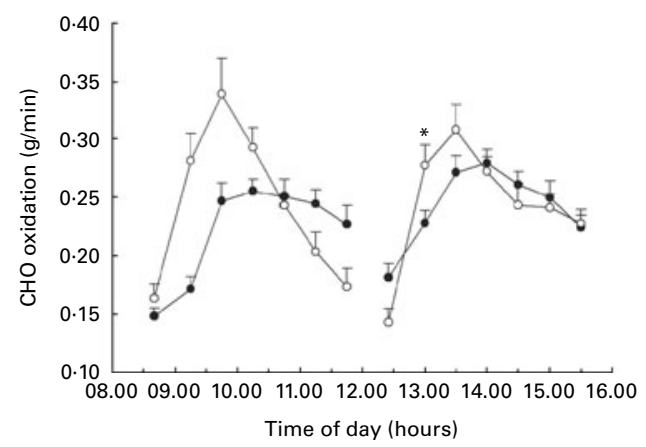

Fig. 2. Time course of TAG concentrations after the intake of (a) trehalose (TRE, --$) v$. glucose (GLUC, $-0-$ ) and (b) isomaltulose (IMU, $-\bullet$ ) v. sucrose (SUC, - O-). Time course of fat oxidation after the intake of (c) TRE $v$. GLUC and (d) IMU v. SUC. Time course of carbohydrate (CHO) oxidation after the intake of (e) TRE $v$. GLUC and (f) IMU $v$. SUC. Values are means, with standard errors of the mean represented by vertical bars $\left(n\right.$ 10). ${ }^{*}$ Mean values were significantly different $(P<0.05)$.

responses following the ingestion of different $\mathrm{CHO}$ after an overnight fast (breakfast), there was a clearly attenuated rise in peak plasma GLUC concentration after the ingestion of IMU compared with SUC and after the ingestion of TRE compared with GLUC (see Fig. 1).

\section{Postprandial TAG concentration}

High plasma TAG concentrations are considered to be risk factors for the development of $\mathrm{CVD}^{(42)}$. Low-glycaemic, lowinsulinaemic $\mathrm{CHO}$ sources may be used to attenuate the postprandial rise in TAG concentrations. However, data show no consensus regarding higher postprandial TAG concentrations following the ingestion of fructose ${ }^{(43,44)}$. In the present study, we observed a trend towards reduced TAG concentrations with ingestion of IMU in combination with a mixed meal compared with SUC ingestion, whereas no such differences were observed for TRE. In contrast, in healthy, overweight subjects, TRE resulted in reduced TAG concentrations during breakfast ${ }^{(20)}$. This discrepancy could be explained by the higher age of the subjects in the present study. Animal as well as human studies generally observed more pronounced effects in younger subjects ${ }^{(45,46)}$.

A limitation of the present study is that the number of subjects is rather small. We cannot rule out sex differences, although the cross-over design limits inter-individual variation. The set-up of the study provides a proof of principle on the impact of TRE and IMU in the breakfast setting and under more physiological conditions where the drink is consumed in combination with a mixed meal. Further studies are warranted to investigate the overall response and physiological significance of the observed differences. 
In conclusion, ingestion of TRE and IMU results in an attenuated postprandial rise in plasma GLUC and insulin concentrations when compared with GLU and SUC, respectively. Co-ingestion of IMU with a mixed meal resulted in an attenuated decline in plasma NEFA concentrations and postprandial fat oxidation rate when compared with SUC, which may reduce ectopic fat accumulation and improve insulin sensitivity. Thus, exchanging SUC for IMU may be favourable to prevent metabolic disturbances, thereby potentially slowing down the progression to type 2 diabetes. More studies are needed to determine the long-term effects of exchanging rapid for more slowly digestible sugars on body-weight control and the prevention of type 2 diabetes in subjects with IGT.

\section{Acknowledgements}

This study was supported by an unrestricted research grant from Cargill R\&D Center Europe, Vilvoorde, Belgium. The authors thank Jos Stegen for expert technical assistance. E. E. B. and F. B. designed the experiment. J. G. P. v. C. executed the experiment, collected the data and wrote the manuscript. L. J. C. v. L. and F. B. read the manuscript and contributed to the discussion. E. E. B. supervised the project, read the manuscript and contributed to the discussion. None of the authors had any financial or personal interest in any company or organisation sponsoring the research.

\section{References}

1. Zimmet P, Alberti KG \& Shaw J (2001) Global and societal implications of the diabetes epidemic. Nature 414, 782-787.

2. DECODE Study Group \& tEDEG (2001) Glucose tolerance and cardiovascular mortality: comparison of fasting and 2-hour diagnostic criteria. Arch Intern Med 161, 397-405.

3. Knowler WC, Barrett-Connor E, Fowler SE, et al. (2002) Reduction in the incidence of type 2 diabetes with lifestyle intervention or metformin. $N$ Engl J Med 346, 393-403.

4. Unwin N, Shaw J, Zimmet P, et al. (2002) Impaired glucose tolerance and impaired fasting glycaemia: the current status on definition and intervention. Diabet Med 19, 708-723.

5. Roumen C, Corpeleijn E, Feskens EJ, et al. (2008) Impact of 3 -year lifestyle intervention on postprandial glucose metabolism: the SLIM study. Diabet Med 25, 597-605.

6. Brand-Miller JC (2004) Postprandial glycemia, glycemic index, and the prevention of type 2 diabetes. Am J Clin Nutr 80, 243-244.

7. Livesey G (2005) Low-glycaemic diets and health: implications for obesity. Proc Nutr Soc 64, 105-113.

8. Ludwig DS (2002) The glycemic index: physiological mechanisms relating to obesity, diabetes, and cardiovascular disease. JAMA 287, 2414-2423.

9. Brand-Miller J, Hayne S, Petocz P, et al. (2003) Low-glycemic index diets in the management of diabetes: a meta-analysis of randomized controlled trials. Diabetes Care 26, 2261-2267.

10. Larsen TM, Dalskov SM, van Baak M, et al. (2010) Diets with high or low protein content and glycemic index for weightloss maintenance. N Engl J Med 363, 2102-2113.

11. Chong MF, Fielding BA \& Frayn KN (2007) Mechanisms for the acute effect of fructose on postprandial lipemia. $A m J$ Clin Nutr 85, 1511-1520.
12. Swanson JE, Laine DC, Thomas W, et al. (1992) Metabolic effects of dietary fructose in healthy subjects. Am J Clin Nutr 55, 851-856.

13. Bantle JP, Raatz SK, Thomas W, et al. (2000) Effects of dietary fructose on plasma lipids in healthy subjects. Am J Clin Nutr 72, $1128-1134$

14. Bossetti BM, Kocher LM, Moranz JF, et al. (1984) The effects of physiologic amounts of simple sugars on lipoprotein, glucose, and insulin levels in normal subjects. Diabetes Care $\mathbf{7}$, 309-312.

15. Brand-Miller JC, Holt SH, Pawlak DB, et al. (2002) Glycemic index and obesity. Am J Clin Nutr 76, 281S-285S.

16. Pawlak DB, Kushner JA \& Ludwig DS (2004) Effects of dietary glycaemic index on adiposity, glucose homoeostasis, and plasma lipids in animals. Lancet 364, 778-785.

17. Scribner KB, Pawlak DB, Aubin CM, et al. (2008) Long-term effects of dietary glycemic index on adiposity, energy metabolism, and physical activity in mice. Am J Physiol Endocrinol Metab 295, E1126-E1131.

18. Isken F, Klaus S, Petzke KJ, et al. (2010) Impairment of fat oxidation under high- vs. low-glycemic index diet occurs before the development of an obese phenotype. Am J Physiol Endocrinol Metab 298, E287-E295.

19. van Can JG, Ijzerman TH, van Loon LJ, et al. (2009) Reduced glycaemic and insulinaemic responses following isomaltulose ingestion: implications for postprandial substrate use. Br J Nutr 102, 1408-1413.

20. van Can JG, Ijzerman TH, van Loon LJ, et al. (2009) Reduced glycaemic and insulinaemic responses following trehalose ingestion: implications for postprandial substrate use. $\mathrm{BrJ}$ Nutr 102, 1395-1399.

21. Stevenson EJ, Thelwall PE, Thomas K, et al. (2009) Dietary glycemic index influences lipid oxidation but not muscle or liver glycogen oxidation during exercise. Am J Physiol 296, E1140-E1147.

22. Corpeleijn E, Mensink M, Kooi ME, et al. (2008) Impaired skeletal muscle substrate oxidation in glucose-intolerant men improves after weight loss. Obesity (Silver Spring) 16, 1025-1032.

23. Mensink M, Blaak EE, van Baak MA, et al. (2001) Plasma free fatty acid uptake and oxidation are already diminished in subjects at high risk for developing type 2 diabetes. Diabetes 50, 2548-2554

24. Adriaens MP, Schoffelen PF \& Westerterp KR (2003) Intraindividual variation of basal metabolic rate and the influence of daily habitual physical activity before testing. BrJ Nutr $\mathbf{9 0}$, 419-423.

25. Schoffelen PF, Westerterp KR, Saris WH, et al. (1997) A dualrespiration chamber system with automated calibration. J Appl Physiol 83, 2064-2072.

26. Weir JB (1949) New methods for calculating metabolic rate with special reference to protein metabolism. I Physiol 109, $1-9$.

27. Frayn KN (1983) Calculation of substrate oxidation rates in vivo from gaseous exchange. $J$ Appl Physiol 55, 628-634.

28. Harris J \& Benedict F (1919) A Biometric Study of Basal Metabolism in Man. Washington, DC: Carnegie Institute of Washington.

29. Richards AB, Krakowka S, Dexter LB, et al. (2002) Trehalose: a review of properties, history of use and human tolerance, and results of multiple safety studies. Food Chem Toxicol $\mathbf{4 0}$, 871-898.

30. Dahlqvist A (1962) Specificity of the human intestinal disaccharidases and implications for hereditary disaccharide intolerance. J Clin Invest 41, 463-470. 
31. Lina BA, Jonker D \& Kozianowski G (2002) Isomaltulose (palatinose): a review of biological and toxicological studies. Food Chem Toxicol 40, 1375-1381.

32. Wolever TM \& Mehling C (2003) Long-term effect of varying the source or amount of dietary carbohydrate on postprandial plasma glucose, insulin, triacylglycerol, and free fatty acid concentrations in subjects with impaired glucose tolerance. Am J Clin Nutr 77, 612-621.

33. Sato K, Arai H, Mizuno A, et al. (2007) Dietary palatinose and oleic acid ameliorate disorders of glucose and lipid metabolism in Zucker fatty rats. J Nutr 137, 1908-1915.

34. Arai H, Mizuno A, Sakuma M, et al. (2007) Effects of a palatinose-based liquid diet (Inslow) on glycemic control and the second-meal effect in healthy men. Metabolism 56, 115-121.

35. Flatt JP (1996) Carbohydrate balance and body-weight regulation. Proc Nutr Soc 55, 449-465.

36. Jenkins DJ, Wolever TM, Ocana AM, et al. (1990) Metabolic effects of reducing rate of glucose ingestion by single bolus versus continuous sipping. Diabetes 39, 775-781.

37. Bays H, Mandarino L \& DeFronzo RA (2004) Role of the adipocyte, free fatty acids, and ectopic fat in pathogenesis of type 2 diabetes mellitus: peroxisomal proliferator-activated receptor agonists provide a rational therapeutic approach. J Clin Endocrinol Metab 89, 463-478.

38. Dahlqvist A \& Thomson DL (1963) The digestion and absorption of maltose and trehalose by the intact rat. Acta Physiol Scand 59, 111-125.

39. Dahlqvist A, Auricchio S, Semenza G, et al. (1963) Human intestinal disaccharidases and hereditary disaccharide intolerance. The hydrolysis of sucrose, isomaltose, palatinose (isomaltulose), and a 1,6-alpha-oligosaccharide (isomalto-oligosaccharide) preparation. J Clin Invest $\mathbf{4 2}$, $556-562$

40. Kawai K, Okuda Y \& Yamashita K (1985) Changes in blood glucose and insulin after an oral palatinose administration in normal subjects. Endocrinol Jpn 32, 933-936.

41. Jentjens RL \& Jeukendrup AE (2003) Effects of pre-exercise ingestion of trehalose, galactose and glucose on subsequent metabolism and cycling performance. Eur J Appl Physiol 88, 459-465.

42. Sparks JD \& Sparks CE (1994) Insulin regulation of triacylglycerol-rich lipoprotein synthesis and secretion. Biochim Biophys Acta 1215, 9-32.

43. Bouche C, Rizkalla SW, Luo J, et al. (2002) Five-week, lowglycemic index diet decreases total fat mass and improves plasma lipid profile in moderately overweight nondiabetic men. Diabetes Care 25, 822-828.

44. Teff KL, Elliott SS, Tschop M, et al. (2004) Dietary fructose reduces circulating insulin and leptin, attenuates postprandial suppression of ghrelin, and increases triglycerides in women. J Clin Endocrinol Metab 89, 2963-2972.

45. Isken F, Weickert MO, Tschop MH, et al. (2009) Metabolic effects of diets differing in glycaemic index depend on age and endogenous glucose-dependent insulinotrophic polypeptide in mice. Diabetologia 52, 2159-2168.

46. van Dam RM, Visscher AW, Feskens EJ, et al. (2000) Dietary glycemic index in relation to metabolic risk factors and incidence of coronary heart disease: the Zutphen Elderly Study. Eur J Clin Nutr 54, 726-731. 